Lüttwitz-Putsches im März 1920 im Auto saß, dass der Reisegesellschaft vor Chemnitz das Benzin ausging und sie nach Stuttgart mit der Bahn weiterfahren musste und dass der (in einem anderen Wagen reisende) Büroleiter des Reichspräsidenten für diesen Zigarren mitführte? Häufig gewinnt man den Eindruck, dass Mühlhausen es versäumt hat, Wichtiges von Unwichtigem zu scheiden, und daher einfach alle Informationen präsentiert, die er gesammelt hat. Auf diese Weise entsteht weniger eine Biographie im klassischen Sinne als ein in seinem Materialreichtum eindrucksvolles Nachschlagewerk zur Reichspräsidentschaft Friedrich Eberts, auf das der Spezialist bei Gelegenheit gern zurückgreifen wird.

Cornelius Torp

\title{
Kurt Georg Kiesinger: verständnisvolle Lebensgeschichte
}

\author{
Gassert, Philipp: Kurt Georg Kiesinger 1904 - 1988. Kanzler zwischen den Zeiten, Deutsche \\ Verlags-Anstalt, München 2006, 896 Seiten, € 39,90.
}

Dass kurz nach Amtsantritt der zweiten Großen Koalition das voluminöse Buch des Heidelberger Historikers Philipp Gassert erschien, dürfte ein Zufall sein. Umso lesenswerter ist diese auf breitester Materialbasis aufbauende, spannend geschriebene erste wissenschaftlich fundierte Biographie über den dritten Kanzler der Bundesrepublik und ersten Regierungschef einer Großen Koalition auf Bundesebene, Kurt Georg Kiesinger. Gerade die Höhen und Tiefen seiner Kanzlerschaft, seine Charakterisierung als angeblich entscheidungsschwacher „wandelnder Vermittlungsausschuss“ zwischen den Koalitionspartnern, die Streitigkeiten innerhalb der Union über die Verjährungsdebatte nationalsozialistischer Gräueltaten, die von der SPD nach verheerenden Wahlniederlagen im Jahr 1968 eingeläutete „Strategie des begrenzten Konflikts“ (Horst Ehmke) sollten ein hervorragender Lese- und Lernstoff für die führenden Entscheidungsträger der zweiten Großen Koalition sein.

Gassert spannt den Lebensbogen des 1904 im schwäbischen Ebingen Geborenen von dessen Studien- und Berufsjahre vor und während des Dritten Reiches über Kiesingers „Entnazifizierungsverfahren“, seinen Aufstieg in der Bundes-CDU als glänzender Debattenredner im Bundestag, seine achtjährige erfolgreiche Amtszeit als Ministerpräsident von BadenWürttemberg bis zur Kanzlerschaft. Auch Kiesingers allmählicher Rückzug aus allen politischen Ämtern, die Abfassung der Memoiren, die allerdings die besonders interessanten Phasen als Regierungschef in Stuttgart und Bonn nicht beinhalten, werden ebenso beleuchtet wie die letzten Jahre in Tübingen, wo der Altkanzler am 8. März 1988 verstarb.

Nahezu ein Viertel dieser Habilitationsschrift ist der Auseinandersetzung mit Kiesingers umstrittener Vergangenheit im „Dritten Reich“ gewidmet - eine Vergangenheit, die den Politiker auf allen Stationen seiner Nachkriegskarriere immer wieder einholte. Nach seinem Jurastudium in Tübingen und Berlin trat er 1933 der NSDAP bei, weigerte sich aber nach den Morden an früheren SA-Führern (der so genannte Röhm-Putsch) - unter Inkaufnahme beruflicher Nachteile - dem NS-Rechtswahrerbund beizutreten und eine Stelle im Staatsdienst anzustreben. Seinen Lebensunterhalt verdiente Kiesinger als juristischer Repetitor, von seinen Schülern wegen seiner begnadeten Fähigkeit, komplizierte Sachverhalte klar darzulegen, als „der Meister“ verehrt. Um der Einberufung zum Wehrdienst zu entgehen, ließ er sich durch Vermittlung eines seiner Schüler in das Auswärtige Amt „dienstverpflich- 
ten“, wo er bis zum Stellvertretenden Leiter der Rundfunkpolitischen Abteilung aufstieg. Diese Tätigkeit überschattete seine gesamte Nachkriegskarriere als Politiker. Eine 1944 erfolgte Denunziation, Kiesinger behindere eine antijüdische Propaganda, entlastete ihn in den Augen seiner Kritiker kaum. Allerdings verhalf der von einem Spiegelredakteur gefundene Beleg für diese Denunziation Kiesinger, die Unionsfraktion am Tag der Abstimmung über die Kanzlernominierung zu überzeugen und für seine Kandidatur zu gewinnen. Auch die SPD akzeptierte dieses Entlastungsmaterial. Dagegen stemmten sich einige Intellektuelle wie Karl Jaspers und Günter Grass sowie die linksliberalen Medien weiterhin gegen einen Kanzler mit „brauner Vergangenheit“.

Philipp Gassert weist in seiner Kiesinger-freundlichen Studie überzeugend nach, dass dieser als „Mitläufer“ einzustufen ist, „keinesfalls aber als Anhänger oder Förderer des NS-Regimes" (S. 85), wie dies seine Gegner versuchten. In einem Wiederaufnahmeverfahren gegen den ursprünglichen Spruch der Entnazifizierungskammer im Sommer 1948 wurde Kiesinger in die Gruppe der „Entlasteten“ eingereiht. „Diese Entlastung war im Hinblick auf Kiesingers politische Laufbahn wichtig", so Gassert, denn sie ermöglichte ihm die Berufung zum Landesgeschäftsführer der CDU von Württemberg-Hohenzollern.

Als Mitglied des Deutschen Bundestages trat Kiesinger, der dem Auswärtigen Ausschuss vorsaß, als brillanter Debattenredner und kompromissloser Anhänger von Adenauers Westintegration hervor. Trotz seiner Verehrung für den Patriarchen wurde Kiesinger von diesem nie in ein Ministeramt berufen. Als er 1957 für das Justizressort „gehandelt“ wurde, besaß der Katholik nicht die richtige Konfession; seine Ernennung hätte das konfessionelle Gleichgewicht innerhalb der Unions-Ministerriege beeinträchtigt. Es verwundert nicht, dass der von Adenauer wenig Geförderte 1958 dem Ruf folgte, das Amt des Ministerpräsidenten des von ihm Anfang der fünfziger Jahre mit geschaffenen Südweststaates zu übernehmen. Kiesinger bezeichnete die acht Jahre seiner Tätigkeit in Stuttgart als die glücklichste Zeit seines politischen Lebens. So reformierte er das dortige Bildungswesen, gründete drei Universitäten (Konstanz, Ulm und Mannheim), legte als aufgeschlossener Konservativer die Grundsteine für den erfolgreichen wirtschaftlichen Aufschwung des Landes und bemühte sich ununterbrochen um ein Zusammenwachsen der beiden Landesteile. Dass er auch glänzend zu repräsentieren verstand, belegen die Staatsbesuche von Charles de Gaulle und Königin Elisabeth II. im „Ländle“.

Als Kompromisskandidat, der nicht in die unionsinternen Intrigen gegen den glücklosen Kanzler Ludwig Erhard verstrickt war, gelang es Kiesinger anschließend mit Hilfe von FranzJosef Strauß und dem jungen Helmut Kohl, von den Partnern der Großen Koalition zum Regierungschef gewählt zu werden.

In seiner Bewertung der Großen Koalition kommt Gassert zu der überzeugenden Schlussfolgerung, dass diese „zu den erfolgreichsten Regierungen der Bundesrepublik gehört. Anfang 1969 hatten die Koalitionspartner, mit der signifikanten Ausnahme der Wahlrechtsreform, sämtliche Punkte aus Kiesingers Regierungserklärung vom 13. Dezember 1966 abgearbeitet und darüber hinaus weitere Reformen verwirklicht" (S. 712). Nicht nur stellte sich der wirtschaftliche Aufschwung rasch ein, sondern es gelang den Koalitionären auch, die Einrichtungen des Sozialstaates weiter auszubauen. In der Außenpolitik beschritten beide Partner vorsichtig eine Öffnung nach Osten. Hierbei musste Kiesinger anders als sein Vizekanzler und Außenminister Willy Brandt in starkem Maße auf die Befindlichkeit seiner Parteifreunde Rücksicht nehmen, die eine Abkehr von der „Hallstein-Doktrin“ ablehnten. Die gemeinsame Regierungsarbeit bilanziert Gassert dahingehend, dass „die Große 
Koalition überwiegend zum innenpolitischen Bündnis [wurde], das der Regierung Brandt/ Scheel auf wirtschafts- und sozialpolitischem Gebiet den Weg frei räumte“ (S. 613).

Ein Jahr vor Ende der Legislaturperiode hatten sich die Regierungspartner „auseinandergelebt“. Die Wahl von Justizminister Gustav Heinemann (SPD) mit den Stimmen der FDP zum Staatsoberhaupt ließ neuen Koalitionsüberlegungen breiten Raum. Kohl schildert in seinen Memoiren anschaulich, wie sich Kiesinger am Wahlabend über Stunden hinweg als Wahlsieger wähnte, um dann kurz vor Mitternacht alles zu verlieren. Kiesinger hatte es nicht vermocht, die FDP dazu zu bewegen, eine konservativ-liberale Koalition zu bilden. In seinen „25 Thesen über Kiesinger“ resümiert Gassert: „Kiesinger wurde zum Verlierer eines Koalitionspokers und schied mit Bitterkeit aus einem Amt, von dem er selbstbewusst annahm, dass er es nicht lang genug innegehabt hatte, um seine Vorstellungen umsetzen zu können." (S. 757) Die Probleme, mit denen ein Kanzler im Kabinett einer Großen Koalition zu kämpfen hat, können nicht eindrucksvoller dargelegt werden. Es ist zu hoffen, dass Kanzlerin Angela Merkel besonders diesen Teil der Biographie des dritten Kanzlers aufmerksam zur Kenntnis nimmt.

Udo Kempf

\section{Essayistische Annäherung an die Große Koalition}

Dittberner, Jürgen: Große Koalition - kleine Schritte. Politische Kultur in Deutschland, Logos Verlag, Berlin 2006, 255 Seiten, $€ 23$,-.

Seitdem Deutschland zum zweiten Mal von einer Großen Koalition regiert wird, ist auch in der Politikwissenschaft das Interesse an dieser Regierungskonstellation wieder gestiegen. Aber was lässt sich in einem Buch, das bereits kurz nach der Entstehung der zweiten Großen Koalition erschienen ist, überhaupt Substantielles über sie sagen? Für eine Bewertung der Politik des jetzigen Regierungsbündnisses ist es zu früh; dieses wird man erst am Ende der Wahlperiode angemessen beurteilen können. Also sollten grundlegende Fragen im Zentrum einer Annäherung an das Thema stehen: Was sind die politischen Voraussetzungen für das Zustandekommen Großer Koalitionen, welche Reformpolitiken sind von ihnen zu erwarten, welcher inneren Dynamik unterliegen sie, wie wirken sie sich auf die Orientierungen und Einstellungen der Bürger aus?

Wer sich eine Antwort auf diese Fragen erhofft, wird von dem Buch von Jürgen Dittberner enttäuscht sein. Statt einer systematischen wissenschaftlichen Analyse erwartet den Leser eher eine essayistische Sammlung persönlicher Eindrücke von politischen Entwicklungen in Deutschland. Dies muss kein Nachteil sein, denn auch gelungene Essays können gute Einblicke in die Problemlagen politischer Phänomene vermitteln, aber diesem Anspruch wird das Buch nur an einigen wenigen Stellen gerecht. Es wirkt über weite Strecken zusammengestückelt, enthält viele Redundanzen und ist außerordentlich fragmentarisch und heterogen.

Der Band beginnt mit einem persönlichen Tagebuch der letzten Wochen der rot-grünen Bundesregierung und streift dann in mehreren Kapiteln verschiedene Perioden der deutschen Politik von den Anfängen der Nachkriegszeit bis zur Gegenwart. Dabei fehlt eine innere Struktur, ein Leitfaden, der die Ausführungen in Richtung des Titels „Große Koali- 